\title{
Editorial: Marine Environmental Epigenetics
}

\author{
Jose M. Eirin-Lopez ${ }^{1 *}$ and Hollie Putnam ${ }^{2 *}$ \\ ${ }^{1}$ Biological Sciences, Florida International University, Miami, FL, United States, ${ }^{2}$ Biological Sciences, University of Rhode \\ Island, Kingston, RI, United States
}

Keywords: epigenetics (DNA methylation histone modifications, chromatin remodeling), phenotypic plasticity, ecology, evolution, marine biology

\section{Editorial on the Research Topic}

\section{Marine Environmental Epigenetics}

\section{OPEN ACCESS}

Edited and reviewed by: Ciro Rico,

University of the South Pacific, Fiji

*Correspondence:

Jose M. Eirin-Lopez jeirinlo@fiu.edu

Hollie Putnam

hputnam@uriedu

Specialty section:

This article was submitted to Marine Molecular Biology and Ecology, a section of the journal

Frontiers in Marine Science

Received: 24 March 2021 Accepted: 06 April 2021

Published: 10 May 2021

Citation:

Eirin-Lopez JM and Putnam H (2021) Editorial: Marine Environmental

Epigenetics.

Front. Mar. Sci. 8:685075 doi: 10.3389/fmars.2021.685075
During the last decade, the growth of the epigenetics field has accelerated rapidly, particularly driven by the transition from basic laboratory-focused studies to more applied efforts including fieldwork, experimental, and observational components. This has been possible thanks to the advent of molecular technologies, expanding our abilities to develop epigenetic analyses in nonmodel organisms (and in a cost-effective fashion), as well as to the advancements in knowledge of epigenetic machinery and genetic-epigenetic interactions in ecologically and environmentally relevant taxa. However, the most compelling reason behind such an explosion has been the growing interest in epigenetics by ecologists, evolutionary biologists, environmental scientists, taxonomists, etc., motivated by the role of these mechanisms governing (to a large extent) phenotypic plasticity responses within and across generations. These mechanisms extend far beyond responses to environmental stress (such as pollution and climate change effects) and include developmental effects, life-history traits, pathogenicity, invasiveness, aging, biomarkers, restoration, among others. Such a broad range of effects showcase the fundamental role of epigenetic regulation in transmitting environmental signals to the genome and regulating how the genetic information is subsequently modulated through gene expression (e.g., phenotypic plasticity) to address such signals. Based on these critical aspects and the rate of advancements, we anticipate that the field known as environmental epigenetics will not be slowing down anytime soon.

While the emergence of environmental epigenetic studies is ubiquitous, the impact of global climate change and the Anthropocene in the world's oceans has driven the need to broaden the epigenetic knowledge to diverse marine ecosystems. The marine system provides a suite of very powerful and informative models to study the role of epigenetic modifications regulating gene function across multiple ecologically and economically important taxa. Critically, such studies are laying the foundations for developing solutions that are informed by epigenetic data, notably assisted evolution and development of biomarkers of stress. The relative absence of epigenetic studies on these subjects, along with the many groups actively developing research on this field, led us to propose a session on this topic at the 2019 Aquatic Sciences Meeting of the Association for the Sciences of Limnology and Oceanography (ASLO), held in San Juan, Puerto Rico. The excitement and momentum generated there translated into the present special issue that Frontiers in Marine Science was kind enough to host. With this effort, our goal was to provide a venue where investigators pioneering the field of marine environmental epigenetics could publish their latest research, and also to help establish a solid foundation informing the future of this field. Based on the 37,000 collective views of all papers published in the present special issue at the time of writing this editorial (probably many more now), we believe we have successfully done so! 
The present special issue consists of 13 research papers gathering more than 60 authors from 25 institutions worldwide. The species studied ranged from microalgae to marine mammals, with a particular emphasis on fish and mollusks. Among the different epigenetic mechanisms, DNA methylation is the most widely studied, although several papers discuss other mechanisms such as chromatin modifiers, histones, and small RNAs. Environmental conditions are challenging organisms in a variety of ways, and this special issue illustrates that by including ocean acidification, thermal stress, seasonal variation, pathogen infection, ocean cycles, among many others, as drivers of the present studies.

Starting with marine algae, Zhao et al. provide a comprehensive study addressing the role of proteins involved in chromatin remodeling (Polycomb and Trithorax groups) using the model diatom Phaeodactylum tricornutum. This work provides a genome-wide profiling of histone marks associated with these proteins, and their role in genome function regulation, underscoring the ancestral nature of these mechanisms.

This special issue also includes two papers focused on corals. In the first, Dimond and Roberts investigate changes in the coral Porites astreoides DNA methylation in Belize in response to simulated environmental change via transplantation to a novel common garden environment. Their findings indicate subtle shifts in DNA methylation state reflective of the move to the common garden environment. The positive relationship between epigenetic and genetic features indicates potential heritability, which would be necessary for methylation to be implicated as a potential mechanism underlying trans-generational acclimatization. In the second paper, Rodriguez-Casariego et al. investigate the dynamic nature of epigenetic changes in the coral Acropora cervicornis. They find temporal changes in genome-wide patterns of DNA methylation, the majority of which are linked to seasonal temperature change. These findings highlight the importance of considering seasonality in field studies to precisely determine the extent of epigenetic responses to environmental stressors.

Several papers use mollusks as model systems to study epigenetic responses to ocean acidification (OA). Bogan et al. quantified global DNA methylation and gene expression over time across different OA regimes in the gasteropod pteropod Limacina helicina antarctica, and calculated historical signatures of methylation. They found global DNA methylation responded to low $\mathrm{pH}$ and linkage between downregulated genes and high values of calculated methylation. These findings support the potential role of DNA methylation in regulating transcriptomic responses to future ocean acidification. In another paper focused on OA, Venkataraman et al. examined the influence of $\mathrm{OA}$ in gonad tissue from the Eastern oyster, Crassostrea virginica, detecting DNA methylation primarily in gene bodies and differentially methylated loci between OA treatments. These results again support the role of DNA methylation in transcriptional control in response to OA, with changes in gonad methylation also indicating the potential for these methylation patterns to be inherited by the offspring. In a complementary paper, Downey-Wall et al. studied DNA methylation in calcifying tissues under OA through time in C. virginica. They found a small number of OA-induced differentially methylated loci, which corresponded with a weak association between OA-induced changes in genome-wide gene body DNA methylation and gene expression and a subtle response in a large number of genes. These results highlight the value of and need for tissue-specific studies of methylation and expression.

Mollusks were also used as models to study the epigenetic response to pathogens. Johnson et al. combined DNA methylation and gene expression in the Eastern oyster to describe responses to infection by the protistan parasite Perkinsus marinus (the cause of the disease commonly known as dermo infection). A network of genes that change in expression in response to infection was discovered, suggesting that gene body DNA methylation is a better predictor of gene expression variation than it is of the overall magnitude of gene expression.

In a more derived marine deuterostome system, Strader et al. explored the epigenetic basis of intra- and inter-generational plasticity in the purple sea urchin by examining relationships between changes in DNA methylation, transcription, and phenotype (embryo spicule length). Here, results suggest that different forms of environmentally induced plasticity are observable across different time scales with a role for DNA methylation, but apparent uncoupling between methylation dynamics in rapid transcriptional responses and whole-organism traits.

Fishes were also well-represented taxa in the present special issue. Accordingly, Fellous and Shama characterized the evolution of the epigenetic machinery involved in key molecular epigenetic pathways including DNA methylation, histone modifications, macroH2A histone, and miRNA biogenesis/turnover in the threespine stickleback, Gasterosteus aculeatus. Their results support the need for similar studies across a diversity of taxa, in order to support progress in epigenetic aspects of assisted evolution, conservation, aquaculture, fisheries, and climate change-adaptation studies. Beyond the stickleback model, Beemelmanns et al. investigated the potential for thermal stress and hypoxia to trigger epigenetic changes in the Atlantic salmon, Salmo salar. Their examination of a specific suite of genes focused on cellular stress response and metabolism uncovering distinct $\mathrm{CpG}$ methylation profiles for fish exposed to different environmental treatment groups. These results support dynamic associations between CpG methylation and transcript expression, supporting wash-in and wash-out temporal dynamics for short-term and longterm acclimatory changes in response to the environment. Intra-generational plasticity and epigenetic inheritance were assessed by Ryu et al. using the reef fish Acanthochromis polyacanthus as a model system. While their prior work reported evidence for inter-generational plasticity, the results presented in this paper support that distinct genetic toolkits may be used for intra-generational plasticity to ocean warming in the same species collected from different latitudes. Examination of multi-generational epigenetic signatures in fish by, Major et al. found changes in epigenetic state in young silversides, Menidia beryllina, when their parents were exposed to endocrine disrupting chemicals (EDCs). The F1 and some of the F2 generation showed altered methylation in genes 
potentially indicative of EDC response providing data for epigenetic inheritance.

Lastly, a study in marine mammals by Beal et al. helps round up the wide taxonomic reach and broad applicability of epigenetic tools representative of the state of the field and in this present special issue. Here, an epigenetic and non-invasive method for aging bottlenose dolphins is defined, building on the known relationship between chronological age and DNA methylation at specific loci. Given that this tool is minimallyinvasive, can be implemented at a large-scale using skin biopsy samples, and critically is accurate in estimating dolphin age, it presents a valuable approach to generating age data from free-ranging cetacean populations.

Collectively, this special issue highlights the growing body of knowledge in marine environmental epigenetics. It showcases both strong and subtle epigenetic responses, supports a link between DNA methylation, transcription, and phenotypic plasticity, and highlights many of the potential applications of this emerging field. Interestingly, although DNA methylation is currently the most well-studied mechanism, the lack of substantial and direct linkage with transcriptional state provides the broad message that the key for epigenetic regulation likely resides in the synergistic interaction among multiple types of epigenetic mechanisms. Such integrative work, while challenging, will enrich our understanding of the eco-evolutionary consequences of epigenetics and inform its applications in the marine context.
We hope you enjoy the present special issue and that it brings new inspiration for your future research. We look forward to working with this emerging field to advance our understanding of marine environmental epigenetics under a changing climate.

\section{AUTHOR CONTRIBUTIONS}

All authors listed have made a substantial, direct and intellectual contribution to the work, and approved it for publication.

\section{FUNDING}

This work was supported by funding from the US National Science Foundation grants EF-1921465 and OCE, IEP, EPSCoR1756623 to HP and EF-1921402 to JME-L.

Conflict of Interest: The authors declare that the research was conducted in the absence of any commercial or financial relationships that could be construed as a potential conflict of interest.

Copyright $(0) 2021$ Eirin-Lopez and Putnam. This is an open-access article distributed under the terms of the Creative Commons Attribution License (CC BY). The use, distribution or reproduction in other forums is permitted, provided the original author(s) and the copyright owner(s) are credited and that the original publication in this journal is cited, in accordance with accepted academic practice. No use, distribution or reproduction is permitted which does not comply with these terms. 